\title{
On the absolute summability of series by Rieszian means
}

\author{
By J. M. Hyslop, University of Glasgow. \\ (Received 7th May, 1936. Read 6th June, 1936.)
}

§1. Definitions and general remarks.

I begin by recalling the well known definitions for summability by the methods of Cesaro and Riesz.

The series $\Sigma a_{n}$ is said to be summable $(C, k), k>-1$, to the sum $s$ if, as $n \rightarrow \infty$,

where

$$
c_{n}^{(k)}=\frac{A_{n}^{(k)}}{E_{n}^{(k)}} \rightarrow s,
$$

$$
A_{n}^{(k)}=\sum_{\nu=0}^{n} E_{\nu}^{(k)} a_{n-\nu}
$$

and $E_{n}^{(k)}$ is defined formally by the relation

$$
\sum_{0}^{\infty} E_{n}^{(k)} x^{n}=(1-x)^{-k-1} \text {. }
$$

If $\lambda_{0}, \lambda_{1}, \ldots, \lambda_{n}, \ldots, \omega$ are positive numbers such that

$$
0 \leqq \lambda_{0}<\lambda_{1}<\lambda_{2}<\ldots<\lambda_{n} \ldots, \quad \lambda_{\mathrm{v}}<\omega \leqq \lambda_{\mathrm{y}+1},
$$

the series $\Sigma a_{n}$ is said to be summable $\left(R, \lambda_{n}, k\right), k \geqq 0$, to the sum $s$ if, as $\omega$ tends to infinity continuously,

$$
C^{(k)}(\omega)=\frac{A^{(k)}(\omega)}{\omega^{k}} \rightarrow s,
$$

where, for $k>-1, A^{(k)}(\omega)$ is defined to be

$$
\sum_{0}^{N}\left(\omega-\lambda_{n}\right)^{k} a_{n} \text {. }
$$

Kogbetliantz ${ }^{1}$ and Obreschkoff ${ }^{2}$ have given the following definitions for the absolute summability of $\Sigma a_{n}$ by these methods:

1 Kogbetliantz, 7. For theorems on summability $|C, k|$ see also Fekete 2, 3, 4, and Winn 11.

2 Obreschkoff, 8, 9. 
If $a_{n}^{(k)}=c_{n}^{(k)}-c_{n-1}^{(k)}$ and if $\sum a_{n}^{(k)}$ is absolutely convergent, then $\Sigma a_{n}$ is said to be summable $|C, k|$.

If $k>0, a \geqq 0$, and if the integral

$$
\int_{a}^{\infty}\left|\frac{d}{d \omega} C^{(k)}(\omega)\right| d \omega
$$

converges, then $\Sigma a_{n}$ is said to be summable ' $R, \lambda_{n}, k \mid$. Summability $\left|R, \lambda_{n}, k\right|$ is therefore equivalent to the bounded variation of the function $C^{(k)}(\omega)$ in the range $(a, \infty)$.

It is at once obvious that summability $\left|R, \lambda_{n}, k\right|$ implies summability $\left(R, \lambda_{n}, k\right)$, that a similar result is true for summability $\mid C, k$, and that summability $\mid R, \lambda_{n}, 0^{\prime}$ ' and $C, 0 ;$ are each equivalent to the absolute convergence of $\Sigma a_{n}$. Also it has been proved ${ }^{2}$ that summability $\left|R, \lambda_{n}, k\right|$ implies summability $\left|R, \lambda_{n}, k^{\prime}\right|$ for $k^{\prime}>k \geqq 0$, and there is a corresponding theore ${ }^{3}$ for the Cesaro method.

J. M. Whittaker ${ }^{4}$ has also defined absolute summability for the Abel or Poisson method, but this will not be required here.

When discussing the summability $(C, k)$ of special series such as Fourier series or Dirichlet series it has often been found convenient to deal with the Rieszian mean rather than with the Cesaro mean. It is permissible to do so in virtue of the well-known equivalence theorem $^{5}$ between the methods $(C, k)$ and $(R, n, k)$. The object of the present paper is to show that the method $|R, n, k|$ is equivalent to the method $|C, k|$. It seems reasonable to expect that this result will be of some use in dealing with the summability $|C, k|$ of certain particular series ${ }^{6}$.

\section{§2. The equivalence theorems.}

It will be proved first of all that summability $|C, k|$ implies summability $|R, n, k|$. Several lemmas will be required in the course of the proof.

LEMMA 1. If $k>-1, \delta>0$, we have

$$
A^{(k+\delta)}(\omega)=\frac{\Gamma(k+\delta+1)}{\Gamma(k+1) \Gamma(\delta)} \int_{0}^{\omega}(\omega-u)^{\delta-1} A^{(k)}(u) d u .
$$

1 This really constitutes the definition of summability $\left|R, \lambda_{n}, 0\right|$.

2 Obreschkoff 9 . $\quad 3$ Kogbetliantz 7. Whittaker $10 . \quad 5$ Hobson 6, 90-93.

- Some results have been obtained recently concerning the summability $|C, k|$ of Fourier series ; see Bosanquet 1.

i Hardy and Riesz 5, 27. 
This result is proved by Hardy and Riesz for the case $k>0$. To prove it for $k>-1$, substitute for $A^{(k)}(u)$ in terms of $a_{n}$ and interchange the order of summation and integration.

It follows from the lemma that, for $k>0$,

$$
A^{(k)}(\omega)=k \int_{0}^{\omega} A^{(k-1)}(u) d u,
$$

so that $A^{(k)}(\omega)$ may be differentiated as though $k$ were an index.

Lemma 2. If $B^{(k)}(\omega)$ is the Rieszian sum of order $k$ for the series $\Sigma b_{n}=\Sigma n a_{n}$, then, for $k>0$,

$$
\omega^{k+1} \frac{d}{d \omega} C^{(k)}(\omega)=k B^{(k-1)}(\omega)=\frac{d}{d \omega} B^{(k)}(\omega) .
$$

We have, by Lemma 1,

$$
\begin{aligned}
\omega^{k+1} \frac{d}{d \omega} C^{(k)}(\omega) & =\omega \frac{d}{d \omega} A^{(k)}(\omega)-k A^{(k)}(\omega) \\
& =k \omega A^{(k-1)}(\omega)-k A^{(k)}(\omega) \\
& =k \sum_{0}^{N}(\omega-n)^{k-1} n a_{n} \\
& =k B^{(k-1)}(\omega) .
\end{aligned}
$$

The proof of this lemma is valid as it stands for the more general case of summability $\left|R, \lambda_{n}, k\right|$ if $b_{n}=\lambda_{n} a_{n}$.

Lemma 3. We have, for $k>-1$, the formal identities

$$
\begin{aligned}
& \text { (i) } \sum_{0}^{\infty} A_{n}^{(k)} x^{n}=(1-x)^{-k-1} \sum_{0}^{\infty} a_{n} x^{n}, \\
& \text { (ii) } \sum_{0}^{\infty} n E_{n}^{(k)} a_{n}^{(k)} x^{n}=(1-x)^{-k} \sum_{0}^{\infty} n a_{n} x^{n} .
\end{aligned}
$$

The first of these follows at once from the definition of $A_{n}^{(k)}$. The second has been proved by Kogbetliantz'

LемMA $4^{2}$. If $k$ is any real number excepl a negative integer, and if

\section{Kogbetliantz 7.}

$2 \mathrm{I}$ am greatly indebted to $\mathrm{Mr} \mathrm{A}$. E. Ingham for permission to reproduce the proofs of Lemmas 4 and 5 which have been taken from notes of lectures delivered by him in 1930-31. In that course of lectures he gave a proof of the equivalence theorem for summability $(C, k)$ and $(R, n, k)$ which has not been published. The proof of Theorem I of this paper has been influenced to some extent by his proof for the corresponding case of ordinary summability. I have also to thank Mr Ingham for important criticisms on this and the earlier part of the paper. 
$q$ is any positive integer, then there exists a sequence of polynomials $p_{0}(\theta), \ldots, p_{q}(\theta)$, such that, for $n \geqq 1$,

$$
(n+\theta)^{k}=\sum_{r=0}^{q} p_{r}(\theta) E_{n}^{(k-r)}+O\left(n^{k-q-1}\right)
$$

uniformly in $0 \leqq \theta \leqq 1$.

Suppose that $k$ is not an integer. By Taylor's Theorem it is clear that

$$
(n+\theta)^{k}=\sum_{\varepsilon=0}^{q}(-1)^{8} E_{\delta}^{(-k-1)} \theta^{s} n^{k-8}+O\left(n^{k-q-1}\right)
$$

uniformly in $0 \leqq \theta \leqq 1$.

Employing Stirling's Theorem we have

$$
\begin{aligned}
E_{n}^{(k-r)} & =\frac{(k-r+n)(k-r+n-1) \ldots(k-r+1)}{n(n-1) \ldots 3.2 .1} \\
& =\sum_{s=r}^{q} \delta_{r, s} n^{k-8}+O\left(n^{k-q-1}\right),
\end{aligned}
$$

where $r=0,1,2, \ldots, q, \delta_{r, 8}$ is a constant and

$$
\delta_{r, r}=\frac{1}{\Gamma(k-r+1)} \neq 0
$$

since $k$ is not an integer.

It follows that

$$
\begin{aligned}
\sum_{r=0}^{q} p_{r} E_{n}^{(k-r)} & =\sum_{r=0}^{q} \sum_{s=r}^{q} p_{r} \delta_{r, 8} n^{k-8}+O\left(n^{k-q-1}\right) \\
& =\sum_{s=0}^{q} n^{k-8} \sum_{r=0}^{s} p_{r} \delta_{r, 8}+O\left(n^{k-q-1}\right)
\end{aligned}
$$

Obviously we can now determine the polynomials $p_{r}$ from the equations

$$
\sum_{r=0}^{s} p_{r} \delta_{r, s}=(-1)^{8} E_{s}^{(-k-1)} \theta^{s}, \quad(s=0,1,2, \ldots, q) .
$$

If $k$ is zero or a positive integer the same argument gives an exact formula without the $O$ term if we take $q=k$. If $q>k$ the lemma is still true provided $p_{r}=0$ for $r>k$.

Lемма 5. If $0<\theta \leqq \mathrm{J}, k>0, q$ is any positive integer, or zero, and

then

$$
\gamma_{n}(\theta)=\sum_{\nu=0}^{n}(n+\theta-\nu)^{k-1} E_{\nu}^{(-k-1)} \text {, }
$$

$$
\gamma_{n}(\theta)=\delta(\theta) E_{n}^{(-k-1)}+O\left\{\sum_{\nu=0}^{n-1}(\nu+1)^{-k-1}(n-\nu)^{k-q-2}\right\}
$$


where

and $e_{r}$ is a constant.

$$
\delta(\theta)=\theta^{k-1}+\sum_{r=0}^{q} e_{r} \theta^{r},
$$

It is clear that

$$
\sum_{0}^{\infty} \gamma_{n}(\theta) x^{n}=(1-x)^{k} \sum_{0}^{\infty}(n+\theta)^{k-1} x^{n} .
$$

Now, by Lemma 4,

where, for $n \geqq 1$,

$$
(n+\theta)^{k-1}=\sum_{r=0}^{q} p_{r}(\theta) E_{n}^{(k-1-r)}+\beta_{n}(\theta)
$$

$$
\beta_{n}(\theta)=O\left(n^{k-2-q}\right) .
$$

Let $e_{r}$ be defined by the relation

$$
\sum_{r=0}^{q} e_{r} \theta^{r}=-\sum_{r=0}^{q} p_{r}(\theta),
$$

and let $\beta_{0}(\theta)=\delta(\theta)$. Then

$$
\begin{aligned}
\sum_{0}^{\infty} \gamma_{n}(\theta) x^{n} & =(1-x)^{k}\left\{\sum_{0}^{\infty} \sum_{r=0}^{q} p_{r} E_{i n}^{(k-1-r)} x^{n}+\sum_{0}^{\infty} \beta_{n} x^{n}\right\} \\
& =\sum_{r=0}^{q} p_{r}(1-x)^{r}+(1-x)^{k} \sum_{0}^{\infty} \beta_{n} x^{n},
\end{aligned}
$$

and therefore, for $n>q$,

$$
\gamma_{n}(\theta)=\sum_{\nu=0}^{n} E_{\nu}^{(-k-1)} \beta_{n-\nu}
$$

Since $E_{n}^{(-k-1)}=O\left(n^{-k-1}\right)$ the result follows. If $n$ is less than $q$ the lemma is obviously true.

This lemma is slightly more general than that given by Ingham, who only required $k>1$, but the proofs in the two cases are almost identical. When $k>1$ the $\delta(\theta)$ term can be incorporated in the summation term giving

$$
\gamma_{n}(\theta)=O\left\{\sum_{\nu=0}^{n}(\nu+1)^{-k-1}(n-\nu+1)^{k-q-2}\right\} .
$$

This result will be required in the proof of Theorem II.

Theorem I. If $k \geqq 0$ and if the series $\Sigma a_{n}$ is summable $|C, k|$, then it is also summable $|R, n, k|$.

It will sometimes be found convenient to use, in the proofs of the theorems which follow, symbols such as $\underset{0}{X}$ where $X$ is a continuous 
variable. This is to be taken to mean $\sum_{0}^{a}$ where $a=X-1$ or [X] according as $X$ is a positive integer or not. A similar meaning is to be attached to $\sum_{X}^{\infty}$.

The theorem is true when $k=0$ since summability $|R, n, 0|$ and $|C, 0|$ are each equivalent to the absolute convergence of $\Sigma a_{n}$. We shall therefore assume that $k$ is positive.

By Lemmas 2 and 3,

$$
\begin{aligned}
\frac{d}{d \omega} C^{(k)}(\omega) & =k \omega^{-k-1} B^{(k-1)}(\omega) \\
& =k \omega^{-k-1} \sum_{1}^{\omega}(\omega-n)^{k-1} n a_{n} \\
& =k \omega^{-k-1} \sum_{n=1}^{\omega}(\omega-n)^{k-1} \sum_{\nu=1}^{n} E_{n-\nu}^{(-k-1)} \nu E_{\nu}^{(k)} a_{\nu}^{(k)}
\end{aligned}
$$

Let $\omega=N+\theta, 0<\theta \leqq 1$ and let $n-\nu=\mu$. Then, interchanging the orders of summation, we obtain

$$
\frac{d}{d \omega} C^{(k)}(\omega)=k \omega^{-k-1} \sum_{\nu=1}^{\omega} \nu E_{\nu}^{(k)} a_{\nu}^{(k)} \sum_{\mu=0}^{N-\nu}(N+\theta-\nu-\mu)^{k-1} E_{\mu}^{(-k-1)},
$$

and, using the notation of Lemma 5,

where

$$
\begin{aligned}
\int_{1}^{X}\left|\frac{d}{d \omega} C^{(k)}(\omega)\right| d \omega & =O\left\{\int_{1}^{X} \omega^{-k-1} d \omega \sum_{\nu=1}^{\omega} \nu E_{\nu}^{(k)}\left|a_{\nu}^{(k)}\right|\left|\gamma_{n-\nu}(\theta)\right|\right\} \\
& =I_{1}+I_{2},
\end{aligned}
$$

$$
\begin{aligned}
& I_{1}=O\left\{\int_{1}^{X} \omega^{-k-1} d \omega \sum_{\nu=1}^{\omega} \nu E_{\nu}^{(k)}\left|a_{\nu}^{(k)}\right| \sum_{\mu=1}^{N-\nu} \mu^{-k-1}(N+1-\nu-\mu)^{k-q-2}\right\}, \\
& I_{2}=O\left\{\int_{1}^{X} \omega^{-k-1} d \omega \sum_{\nu=1}^{\omega} \nu E_{\nu}^{(k)}\left|a_{\nu}^{(k)}\right|\left|\delta(\theta)_{i}\right| E_{N-\nu}^{(-k-1)} \mid\right\} .
\end{aligned}
$$

Rearranging the orders of summation and integration, and putting $\rho-\nu+1=\mu$, we obtain

$$
\begin{aligned}
I_{1} & =O\left\{\sum_{\nu=1}^{X} \nu E_{\nu}^{(k)}\left|a_{\nu}^{(k)}\right| \sum_{\rho=\nu}^{X}(\rho-\nu+1)^{-k-1} \int_{\rho+1}^{X} \omega^{-k-1}(N-\rho)^{k-q-2} d \omega\right\} \\
& =O\left\{\sum_{\nu=1}^{X} \nu E_{\nu}^{(k)}\left|a_{\nu}^{(k)}\right| \sum_{\rho=\nu}^{X}(\rho-\nu+1)^{-k-1} \rho^{-k-1} \sum_{\sigma=1}^{\infty} \int_{\rho+\sigma}^{\rho+\sigma+1}(N-\rho)^{k-q-2} d \omega\right\} \\
& =O\left\{\sum_{\nu=1}^{X} \nu E_{\nu}^{(k)}\left|a_{\nu}^{(k)}\right| \sum_{\rho=\nu}^{X} \rho^{-k-1}(\rho-\nu+1)^{-k-1} \sum_{\sigma=1}^{\infty} \sigma^{k-q-2}\right\} .
\end{aligned}
$$


Choose $q$ greater than $k-1$. Then

$$
\begin{aligned}
I_{1} & =O\left\{\sum_{\nu=1}^{X} \nu E_{\nu}^{(k)}\left|a_{\nu}^{(k)}\right| \sum_{\rho=\nu}^{X} \rho^{-k-1}(\rho-\nu+1)^{-k-1}\right\} \\
& =O \cdot\left\{\sum_{\nu=1}^{X} \nu^{-k} E_{\nu}^{(k)}\left|a_{\nu}^{(k)}\right| \sum_{\rho=\nu}^{\infty}(\rho-\nu+1)^{-k-1}\right\} \\
& =O\left\{\sum_{\nu=1}^{\infty}\left|a_{\nu}^{(k)}\right|\right\} \\
& =O(1) . \\
& \text { Also } \\
I_{2} & =O\left\{\sum_{\nu=1}^{X} \nu E_{\nu}^{(k)}\left|a_{\nu}^{(k)}\right| \int_{\nu}^{X} \omega^{-k-1}(N-\nu+1)^{-k-1}|\delta(\theta)| d \omega\right\} \\
& =O\left\{\sum_{\nu=1}^{X}\left|a_{\nu}^{(k)}\right| \int_{\nu}^{X}(\omega-N)^{k-1}(N-\nu+1)^{-k-1} d \omega\right\} \\
& =O\left\{\sum_{\nu=1}^{X}\left|a_{\nu}^{(k)}\right| \sum_{\rho=0}^{\infty} \int_{\nu+\rho}^{\nu+\rho+1}(\omega-N)^{k-1}(N-\nu+1)^{-k-1} d \omega\right\} \\
& =O\left\{\sum_{\nu=1}^{X}\left|a_{\nu}^{(k)}\right| \sum_{\rho=0}^{\infty}(\rho+1)^{-k-1} \int_{\nu+\rho}^{\nu+\rho+1}(\omega-\nu-\rho)^{k-1} d \omega\right\} \\
& =O\left\{\sum_{\nu=1}^{\infty}\left|a_{\nu}^{(k)}\right| \sum_{\rho=0}^{\infty}(\rho+1)^{-k-1}\right\} \\
& =O(1) .
\end{aligned}
$$

The theorem is therefore proved.

We require another lemmal in order to prove the converse:

LEMMA 6. If $k$ is a positive integer or zero, $A_{n}^{(k)}$ can be expressed in the form

where $d_{\rho}$ is a constant.

$$
\sum_{\rho=0}^{k} d_{\rho} A^{(k)}(n+\rho / k)
$$

THEOREM II. If $k \geqq 0$ and if $\Sigma a_{n}$ is summable $|R, n, k|$, then it is also summable: $C, k \mid$.

As in Theorem I we take $k$ to be positive. Using Lemmas 3, 6, 1 and 2 we have

$$
\begin{aligned}
n E_{n}^{(k)} a_{n}^{(k)} & =\sum_{\nu=0}^{n} E_{n-\nu}^{(k-1)} b_{\nu} \\
& =\sum_{\nu=0}^{n} E_{n-\nu}^{(k-1)} \sum_{\mu=0}^{\nu} E_{\nu-\mu}^{(-i-2)} B_{\mu}^{(i)} \\
& =\sum_{\rho=0}^{i} d_{\rho} \sum_{\nu=0}^{n} E_{n-\nu}^{(k-1)} \sum_{\mu=0}^{\nu} E_{\nu-\mu}^{(-i-2)} B^{(i)}(\mu+\phi) \\
& =D_{k} \sum_{\rho=0}^{i} d_{\rho} \sum_{\nu=0}^{n} E_{n-\nu}^{(k-1)} \sum_{\mu=0}^{v} E_{\nu-\mu}^{(-i-2)} \int_{0}^{\mu+\phi} \frac{d}{d u}\left\{B^{(k)}(u)\right\} \cdot(\mu+\phi-u)^{i-k} d u,
\end{aligned}
$$

1 Hobson 6, 93. 
where $i$ is some integer greater than $k, \phi=\phi(\rho)=\rho / i$, and

$$
D_{k}=\frac{\Gamma(i+1)}{\Gamma(k+1) \Gamma(1+i-k)} .
$$

Employing Lemma 2 and interchanging the order of the summations and integration we obtain

$n E_{n}^{(k)} a_{n}^{(k)}=D_{k} \sum_{\rho=0}^{i} d_{\rho} \int_{0}^{n} u^{k+1} \frac{d}{d u}\left\{C^{(k)}(u)\right\} d u \sum_{\mu=u-\phi}^{n}(\mu+\phi-u)^{i-k} \sum_{\nu=\mu}^{n} E_{n-\nu}^{(k-1)} E_{\nu-\mu}^{(-i-2)}$.

But

$$
\sum_{\nu=\mu}^{n} E_{n-\nu}^{(k-1)} E_{\nu-\mu}^{(-i-2)}=\sum_{p=0}^{n-\mu} E_{n-\mu-p}^{(k-1)} E_{p}^{(-i-2)}
$$

which is the coefficient of $x^{n-\mu}$ in the expansion of

$$
(1-x)^{-k}(1-x)^{i+1}
$$

This coefficient, by definition, is $E_{n-\mu}^{(k-i-2)}$. Hence $n E_{n}^{(k)}, a_{n}^{(k)}=D_{k} \sum_{\rho=0}^{i} d_{\rho} \int_{0}^{n} u^{k+1} \frac{d}{d u}\left\{C^{(k)}(u)\right\} d u \sum_{\mu=u-\phi}^{n}(\mu+\phi-u)^{i-k} E_{n-\mu}^{(k-i-2)}$.

Divide by $n E_{n}^{(k)}$, take absolute values, sum from 0 to $N$ and apply Lemma 5. Then, since $i>k$,

$$
\begin{aligned}
\sum_{0}^{N}\left|a_{n}^{(k)}\right|=O\left\{\sum_{\rho=0}^{i}\left|d_{\rho}\right| \sum_{0}^{N}(n+1)^{-k-1} \int_{0}^{n} u^{k+1}\left|\frac{d}{d u} C^{(k)}(u)\right| d u\right. \\
\left.\sum_{\mu=u-\phi}^{n}(n-\mu+1)^{k-i-2}(\mu+\phi+1-u)^{i-k-q-1}\right\} .
\end{aligned}
$$

Taking $q=i$ and interchanging the order of the summations and integration we obtain

$$
\begin{aligned}
\sum_{0}^{N}\left|a_{n}^{(k)}\right| & =O\left\{\sum_{\rho=0}^{i}\left|d_{\rho}\right| \int_{0}^{N} u u^{k+1}\left|\frac{d}{d u} C^{(k)}(u)\right| d u\right. \\
& \left.\sum_{\mu=u-\phi}^{N}(\mu+\phi+1-u)^{-k-1} \sum_{n=\mu}^{N}(n+1)^{-k-1}(n-\mu+1)^{k-i-2}\right\} \\
& =O\left\{\sum_{\rho=0}^{i}\left|d_{\rho}\right| \int_{0}^{N} u^{k+1} \frac{d}{d u} C^{(k)}(u) d u \sum_{\mu=u-\phi}^{N}(\mu+\phi+1-u)^{-k-1}(\mu+1)^{-k-1}\right\} \\
& =O\left\{\sum_{\rho=0}^{i}\left|d_{\rho}\right| \int_{0}^{N} u^{k+1} \frac{d}{d u} C^{(k)}(u)^{\mid}(u+1-\phi)^{-k-1} d u\right\} \\
& =O\left\{\int_{0}^{N} \mid \frac{d}{d u} C^{(k)}(u) d u\right\} \\
& =O(1) .
\end{aligned}
$$

The theorem is therefore proved. 


\section{REFERENCES.}

1. L. S. Bosanquet, Journal London Math. Soc., 11 (1936), 11-15.

2. M. Fekete, Math. és termész. ért., 29 (1911), 719-726.

3. M. Fekete, Math. és termész. ért., 32 (1914), 389-425.

4. M. Fekete, Proc. Edinburgh Muth. Soc. (2), 3 (1932-33), 132-134.

5. G. H. Hardy and M. Riesz, The General Theory of Dirichlet Series. (Cambridg Tract No. 18).

6. E. W. Hobson, The Theory of Functions of a Real Variable II (1926).

7. E. Kogbetliantz, Bull. des Sciences Math. (2), 49 (1925), 234-256.

8. N. Obreschkoff, Comptes Rendus, 186 (1928), 215.

9. N. Obreschkotf, Math. Zeitschrift, 30 (1929), 375-386.

10. J. M. Whittaker, Proc. Edinburgh Math. Soc. (2), 2 (1930-31), 1-5.

11. C. E. Winn, Proc. Edinburgh Math. Soc. (2), 3(1932-33), 173.178. 